\title{
Magnetic susceptibility of EuTe/PbTe Heisenberg antiferromagnetic superlattices: experimental and theoretical studies
}

\author{
Lorenzo Bergomi ${ }^{\S}$ \\ Department of Physics, Massachusetts Institute of Technology, Cambridge MA 02139 \\ and CEA, Service de Physique Théorique, 91191 Gif-sur-Yvette Cedex, France \\ James J. Chen \\ Department of Physics, Massachusetts Institute of Technology, Cambridge MA 02139
}

\begin{abstract}
We report results on the temperature dependence of the susceptibilities of a set of MBE-grown short-period EuTe/PbTe antiferromagnetic superlattices having different EuTe layer thicknesses. In-plane and orthogonal susceptibilities have been measured and display a strong anisotropy at low temperature, confirming the occurrence of a magnetic phase transition in the thicker samples, as seen also in neutron diffraction studies. We suggest that dipolar interactions stabilize antiferromagnetic long-range order in an otherwise isotropic system and we present numerical and analytical results for the low-
\end{abstract}

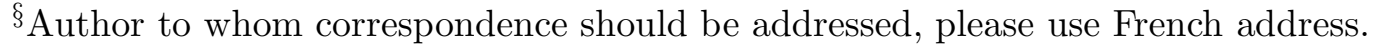
email: bergomi@spht.saclay.cea.fr

${ }^{\ddagger}$ Current address: Lehman Brothers Inc., 3 World Financial Center, New York NY 10285.
} 
temperature orthogonal susceptibility.

Typeset using REVTEX 


\section{INTRODUCTION}

Magnetic films and multilayers have been a subject of intense study since they provide experimental realizations for various two-dimensional (2D) magnetic models. 1 . 2 Most of the recent literature has focused on metallic magnetic structures. However, insulating antiferromagnetic structures provide an opportunity to study magnetic long-range order in $2 \mathrm{D}$ layered systems of localized spins. Among those, the EuTe/PbTe superlattice (SL) structures are of special interest since only one of the two components, EuTe, is magnetic.

Bulk EuTe is a type-II antiferromagnet of the family of Europium chalcogenides, with the structure of $\mathrm{NaCl}$. Its magnetic moments arise from the strongly localized $4 f$ electrons of the $\mathrm{Eu}^{2+}$ atoms which are in a symmetric ${ }^{8} S_{7 / 2}$ ground state. Thus, the europium chalcogenides have long been considered ideal realizations of isotropic Heisenberg models. Antiferromagnetic resonance experiments have confirmed that, once dipolar interactions are taken into account, the residual anisotropy in EuTe is negligible. temperature $\left(T_{N}\right)$ of $9.8 \mathrm{~K}$. Below $T_{N}$, spins belonging to a single (111) plane are parallel but antiparallel to spins in adjacent (111) planes. The magnetic properties of EuTe are described by a Heisenberg Hamiltonian with two exchange constants: $J_{1}$ (nearest neighbors, ferromagnetic) and $J_{2}$ (next-nearest neighbors, antiferromagnetic):

$$
\mathcal{H}_{e x}=J_{1} \sum_{n n} \mathbf{S}_{i} \cdot \mathbf{S}_{j}+J_{2} \sum_{n n n} \mathbf{S}_{i} \cdot \mathbf{S}_{j}
$$

The $\mathbf{S}_{i}$ vectors denote $\mathrm{Eu}^{2+}$ spins, which have magnitude $7 / 2 . J_{1}$ and $J_{2}$ are not known very precisely; currently accepted values are: $J_{1} / k_{B}=-0.04 \pm 0.01 K$ and $J_{2} / k_{B}=0.15 \pm 0.01 K$, where $k_{B}$ is the Boltzmann constant 目目

Although the Hamiltonian in eq. (1) has full rotational symmetry, neutron diffraction experiments show that the spins lie in (111) planes. accounted for by adding dipole-dipole interactions to the exchange Hamiltonian in eq. (11). 10

In this article we present experimental and theoretical studies of the susceptibilities of short-period EuTe/PbTe SL's. We use the notation $\operatorname{EuTe}(\xi) / \mathrm{PbTe}(\eta)$ to denote a SL 
structure whose period consists of $\xi$ monolayers of EuTe and $\eta$ monolayers of PbTe. Each sample used in the present work consisted of 400 such periods and was prepared so that $\eta=3 \xi$. Since samples are grown in the (111) direction, the structure within the EuTe monolayers is that of a triangular lattice and the monolayers are stacked according to the ABC sequence. Section II describes our samples and presents results for their temperaturedependent zero-field susceptibilities. Section III presents a discussion of the experimental results, then Section IV presents a mean-field determination of the order parameter, a MonteCarlo simulation of the susceptibilities of an $\operatorname{EuTe}(3) / \operatorname{PbTe}(9)$ sample, and a calculation of the out-of-plane susceptibility at low temperature. Section V summarizes our findings.

\section{EXPERIMENTAL RESULTS}

\section{A. Sample Characterization}

Experiments were conducted on SL samples of $\operatorname{EuTe}(\xi) / \mathrm{PbTe}(3 \xi)$ for $1 \leq \xi \leq 7$. In each sample the SL stack was grown on a $3000 \AA$ PbTe (111) oriented buffer layer, itself grown on a $\mathrm{BaF}_{2}$ (111) substrate. A $500 \AA$ PbTe cap layer was used to prevent oxidation of the highly reactive EuTe. Details of the MBE growth process have been published elsewhere.11-13 The SL's have approximately square wave composition modulation, as reflected by the multiple narrow SL peaks of the high-resolution x-ray diffraction data. 14 Electron spin resonance (ESR) experiments show very little interdiffusion at the EuTe-PbTe interface 15 Furthermore, careful in situ scanning tunneling microscopy (STM) investigations have shown that the PbTe and EuTe heterointerfaces are quite smooth on a length scale of at least $200 \AA$, with imperfections strictly limited to single monolayer steps 17 The ex situ cross-sectional TEM images also exhibit smooth $\mathrm{PbTe}$ and EuTe interfaces.18

The magnetic properties of the SL's used have been studied previously by SQUID

magnetometry, 14 and elastic neutron scattering 19 Magnetic hysteresis curves and neutron diffraction spectra taken at $1.8 \mathrm{~K}$ and $4.2 \mathrm{~K}$ show that for all samples with $\xi \geq 3$ a transition 
to a low-temperature ordered phase takes place at $T_{N} \geq 4.2 \mathrm{~K}$, the order being that of a type II antiferromagnet, i.e. identical to that of bulk EuTe. Since the MBE samples are grown along the (111) direction, this implies that the spins in each EuTe monolayer order ferromagnetically and are antiparallel to those in neighboring monolayers. Moreover, the spins lie within the EuTe monolayers. Static magnetization measurements taken parallel to the SL plane show no detectable in-plane anisotropy 16

\section{B. Experimental Conditions}

Our susceptiblility measurements used a Quantum Design (MPMS5) AC susceptometer. Susceptibilities were measured in two geometries : $\chi_{i n}$ was measured with the AC probing field $h$ parallel to the SL plane, $\chi_{\text {out }}$ was measured with $h$ orthogonal to the SL plane (see Fig. 1).

For each sample, $\chi_{\text {in }}$ and $\chi_{\text {out }}$ have been measured as a function of temperature from 15 $\mathrm{K}$ in the paramagnetic region to below the transition temperature, in a nominal zero external static magnetic field (i.e., less than 2 Gauss) with a $20 \mathrm{~Hz}$ AC probing field of 4 Gauss. Since the probing field is small, diamagnetic contributions from the $\mathrm{BaF}_{2}$ substrate and the $\mathrm{PbTe}$ buffer layer can safely be ignored. A study of the dependence of the susceptibility on $h$ and on the $\mathrm{AC}$ frequency indicates that our measurements are always in the linear regime of the static susceptibility.

\section{Experimental Susceptibilities}

Figure 2 displays the temperature dependence of the in-plane and ou-of-plane magnetic

susceptibilities $\chi_{\text {in }}$ and $\chi_{\text {out }}$ for samples $\operatorname{EuTe}(2) / \mathrm{PbTe}(6)$ through $\operatorname{EuTe}(7) / \mathrm{PbTe}(21)$. The experimental susceptibilities have been normalized by the number of $\mathrm{Eu}^{2+}$ spins, determined using high temperature susceptibility data; no demagnetizing corrections have been applied to the data. In the following we will be mostly interested in $\chi_{\text {out }}$, whose magnitude changes 
little below the temperature at which $\chi_{\text {out }}$ and $\chi_{\text {in }}$ separate. Since there is no observable difference in $\chi_{\text {out }}$ and $\chi_{\text {in }}$ above this temperature we can assume that demagnetizing corrections are not significant.

For all samples with $\xi>1$ a plot of $\chi T^{2}$ versus $T$ showed that, as the temperature is lowered, the Curie behavior of $\chi_{i n}$ and $\chi_{\text {out }}$ persists until they separate at a temperature $T_{s} .16$ Fig. 4 shows such a plot for sample $\operatorname{EuTe}(3) \operatorname{PbTe}(9)$. Generically, below the point of separation a very anisotropic behavior of the susceptibility is observed, with $\chi_{\text {out }} \leq \chi_{\text {in }}$, an unusual feature. Furthermore, while $\chi_{\text {in }}$ displays a peak, $\chi_{\text {out }}$ has a very mild temperature dependence below $T_{s}$. In addition, the magnitude of $\chi_{\text {out }}$ at low temperature, of the order of $1.10^{-24} \mathrm{emu} / \mathrm{spin}$, has little dependence on the thickness of the EuTe layer. Since we do not have other experimental data (e.g. specific heat) that would allow us to define more precisely the transition temperature $T_{N}$, we assumed that it lies in between $T_{s}$ and the temperature at which $\chi_{i n}$ is maximum. $T_{N}$ increases with the thickness of the EuTe layer, as expected, and reaches values higher than that for bulk EuTe for samples with $\xi \geq 5$, an unexpected result. A possible explanation could be that coupling constants have values different from the bulk ones, a point developed further in section $\nabla$. We comment now on samples that show non-generic behavior.

\section{1. $\operatorname{EuTe}(1) / \operatorname{PbTe}(3)$}

$\chi_{\text {in }}$ and $\chi_{\text {out }}$ as well as $\chi_{\text {in }} T^{2}$ and $\chi_{\text {out }} T^{2}$ have been measured for $T>1.8 \mathrm{~K}$. They are plotted in Fig. 3. One sees that in this temperature regime sample $\operatorname{EuTe}(1) / \mathrm{PbTe}(3)$ remains in a paramagnetic phase $: \chi_{\text {in }}$ and $\chi_{\text {out }}$ coincide and exhibit a Curie behavior. The absence of a transition in this temperature range can be understood by noting that in a monolayer geometry the $J_{2}$ exchange coupling is not present and the energy scale is set by the nearest-neighbor exchange coupling only, which is very small. We expect however a transition at a lower temperature. 


\section{2. $\operatorname{EuTe}(2) / \operatorname{PbTe}(6)$}

Elastic neutron scattering spectra show no long-range order at $4.2 \mathrm{~K}$ but they do show a peak corresponding to type II antiferromagnetic ordering at $1.8 \mathrm{~K}$. Thus the broad maximum in $\chi_{i n}$ does not signal a transition from the paramagnetic to the antiferromagnetic phase.

\section{3. $\operatorname{EuTe}(6) / P b T e(18)$}

The sharp drop in $\chi_{\text {out }}$ around $5 \mathrm{~K}$ is reproducible. However we only had one $\mathrm{EuTe}(6) / \mathrm{PbTe}(18)$ sample. $\mathrm{EuTe}(6) / \mathrm{PbTe}(18)$ is the only sample in our series that displays this feature, as yet unexplained.

\section{DISCUSSION}

A qualitatively different behavior for $\chi_{i n}$ is expected a priori, depending on whether the number of EuTe monolayers per SL period is odd or even. In the first case, each period should behave as a ferromagnet and there should be a peak in $\chi_{i n}$ whereas in the latter case each period should behave as an antiferromagnet and we should see a smooth maximum. Although we use integers $\xi$ and $\eta$ to label our samples, the average thickness of, say, the EuTe layer in an actual SL period as determined by X-ray scattering is fractional because of the interface structure so that the odd/even effect is in fact expected to be blurred.14

In our view, the main issues raised by our data is the existence of a phase transition in samples with $\xi \geq 3$ at temperatures comparable to the bulk $\mathrm{T}_{N}$. As mentioned above, neutron diffraction spectra unambiguously demonstrate that these samples have a lowtemperature ordered phase. Also, anisotropy in the Hamiltonian for $\mathrm{Eu}^{2+}$ spins is negligible, so that our samples can be considered as representing a model 2D Heisenberg system. In such

a system, with isotropic exchange couplings, the transition should occur at $\mathrm{T}=0 \mathrm{~K} 20$ The Hamiltonian is however incomplete and we need to supplement the exchange couplings with dipole-dipole interactions. Although the dipole-dipole coupling may be weak, it breaks the 
rotational symmetry and is long-range, which prevents application of the Mermin-Wagner theorem.2 Dipolar couplings are known to have large effects in metallic thin films, in which they compete with uniaxial anisotropy.21,22

Early work 23 has demonstrated the possibility of a phase transition driven by dipolar interactions in a 2D isotropic spin system. More recent work has investigated the dependence of $T_{N}$ on the magnitude of the dipolar coupling.25. 27. It is our hypothesis that dipolar interactions are responsible for stabilizing magnetic long-range order in EuTe/PbTe SL's. In the next section we discuss some implications of this hypothesis.

The observation that $\chi_{\text {out }} \leq \chi_{\text {in }}$ for all samples can be understood by noting that there are actually three pertinent susceptibilities in our system : $\chi_{\|}$, along the direction of the order parameter in the SL plane, $\chi_{\perp}$, in the SL plane, but orthogonal to the direction of the order parameter, and $\chi_{\text {out }}$, orthogonal to the SL plane. In our case, $\chi_{\perp}$ and $\chi_{\text {out }}$ will be different because of dipolar interactions, and $\chi_{\text {out }}$ will be smaller than $\chi_{\perp}$. Usually one has $\chi_{\|} \leq \chi_{\perp}$, and in our case we expect $\chi_{\|} \leq \chi_{\text {out }} \leq \chi_{\perp}$. The assumption that in each atomic layer the spins belong to domains with random orientations yields : $\chi_{i n}=\frac{1}{2}\left(\chi_{\|}+\chi_{\perp}\right)$. It is thus possible for $\chi_{\text {out }}$ to be smaller than $\chi_{i n}$.

\section{THEORY}

In this section, we use the following Hamiltonian to describe the interaction of $\mathrm{Eu}^{2+}$ spins:

$$
\mathcal{H}=J_{1} \sum_{n n} \mathbf{S}_{i} \cdot \mathbf{S}_{j}+J_{2} \sum_{n n n} \mathbf{S}_{i} \cdot \mathbf{S}_{j}+\sum_{i j, \alpha \beta} Q_{\alpha \beta}\left(\mathbf{r}_{j}-\mathbf{r}_{i}\right) S_{i}^{\alpha} S_{j}^{\beta},
$$

where the third sum runs over all sites $i$ and $j$ in the SL and on spin components $\alpha, \beta$. Here $Q_{\alpha \beta}\left(\mathbf{r}_{j}-\mathbf{r}_{i}\right)$ is the dipolar tensor which reads:

$$
Q_{\alpha \beta}\left(\mathbf{r}_{j}-\mathbf{r}_{i}\right)=\frac{\left(g \mu_{b}\right)^{2}}{2}\left(\frac{\delta^{\alpha \beta}}{r_{i j}^{3}}-3 \frac{r_{i j}^{\alpha} r_{i j}^{\beta}}{r_{i j}^{5}}\right) .
$$

In this expression, $\mu_{b}$ is the Bohr magneton and $g$ is the Landé $g$ factor which we will take equal to 2. Throughout this section we will approximate the spins $\mathbf{S}_{i}$ by classical vectors. 
This is justified by the large magnitude $S=\frac{7}{2}$ of the $\mathrm{Eu}^{2+}$ spins and the fact that we don't expect quantum effects in the temperature range we will be considering. The structure within the EuTe layers is that of a triangular lattice and the layers are stacked according to the ABC sequence. We will take the $x$ and $y$ axes in the plane of the layer and the $z$ axis orthogonal to the layer plane.

\section{A. Orders of magnitude}

Let us denote by $E_{\|}$and $E_{\perp}$ the dipolar energies per spin of an EuTe monolayer, assuming the spins are ferromagnetically aligned either in the layer plane or orthogonal to the layer plane. $E_{\perp}$ is given by :

$$
E_{\perp}=\sum_{i} Q_{z z}\left(\mathbf{r}_{i}\right)=\frac{\left(g \mu_{b} S\right)^{2}}{2} \sum_{i} \frac{1}{r_{i}^{3}}
$$

where the sums run on the sites of a single layer. $E_{\|}$is given by :

$$
E_{\|}=\sum_{i} Q_{x x}\left(\mathbf{r}_{i}\right)=-\frac{\left(g \mu_{b} S\right)^{2}}{4} \sum_{i} \frac{1}{r_{i}^{3}}
$$

where we have used the fact that $Q$ is traceless and rotationally invariant in the $x y$ plane. Notice that $E_{\|}<0$, which favors in-plane alignment, as observed in neutron diffraction experiments. Using the value of $\sum_{i} \frac{1}{r_{i}^{3}}=11.035 / a^{3}$ for a triangular lattice and the value of the in-plane lattice parameter $a=4.6 \AA$ taken from $\mathrm{X}$-ray data, we get $E_{\|}=-0.86 \mathrm{~K}$ and $E_{\perp}=1.72 \mathrm{~K}$, to be compared with the exchange energies $J_{1} S^{2}=-0.5 \mathrm{~K}, J_{2} S^{2}=1.8 \mathrm{~K}$ and the average exchange energy per spin $E_{e x}=-6 J_{2} S^{2}=11 \mathrm{~K}$, which we have estimated using bulk EuTe values for $J_{1}, J_{2}$.

Next we estimate the size of the interlayer dipolar energies. To this end we have used

Ewald summation techniques that allow one to rewrite $\frac{1}{r^{3}}$ sums as fast-converging series.2328 Let us consider two neighboring EuTe monolayers (1) and (2) a distance $h$ apart. Let $\mathbf{r}$ be the vector joining a lattice site in layer (1) to a lattice site in layer (2). Assuming that the spins in layer (1) and (2) are all ferromagnetically aligned but with opposite directions 
depending on which layer they belong to, the interaction energy of a spin in layer (1) with all spins in layer (2) is:

$$
E=-\frac{\left(g \mu_{b} S\right)^{2}}{2} \frac{2 \pi}{\mathcal{A}} \sum_{\mathbf{G}} \frac{G_{x}^{2}}{G} e^{-h G} \cos (\mathbf{G} . \mathbf{r})
$$

where the sum runs over all reciprocal lattice vectors $\mathbf{G}$, and $G$ and $G_{x}$ are, respectively, the modulus and the $x$-component of $\mathbf{G}$ and $\mathcal{A}$ is the area of the triangular lattice unit cell in the layer. We thus get :

$$
E=0.1014 \frac{\left(g \mu_{b} S\right)^{2}}{a^{3}}=0.0317 \mathrm{~K}
$$

This energy is much smaller than the intralayer energy $E_{\|}$. If the spin density in the layer were uniform, no field would be created outside the layer and this energy would be 0 . The very existence of a lattice structure within the layer makes it finite. Furthermore, the interaction energy is expected to decay fast as the distance from the layer becomes larger than the in-plane lattice constant. For instance, at a distance two layers away, this energy is $-8.810^{-5} \mathrm{~K}$, and three layers away it is $4.710^{-7} \mathrm{~K}$.

As a result, we can safely discard all interlayer couplings as well as interperiod couplings, which couple spins belonging to different SL periods. Although small, the intralayer coupling has to be retained for the reasons mentioned in section III].

\section{B. Mean Field analysis}

We now turn to a Mean Field treatment of our problem. Our aim here is to identify the order parameter for the phase transition rather than find the expression for $T_{N}$.. We consider a single period of a SL which consists of $N$ EuTe monolayers. The spins are labelled with two indices: $i$ denotes their position in a layer, $n$ the layer to which they belong. We rewrite the Hamiltonian as:

$$
\mathcal{H}=\sum_{i j n m, \alpha \beta} H_{i n \alpha, j m \beta} S_{i n}^{\alpha} S_{j m}^{\beta}
$$

where the matrix $H$ is defined by: 


$$
H_{i n \alpha, j m \beta}=\delta^{\alpha \beta} J\left(\mathbf{r}_{j}-\mathbf{r}_{i}, m-n\right)+\delta^{n m} Q_{\alpha \beta}\left(\mathbf{r}_{j}-\mathbf{r}_{i}\right)
$$

in which $\mathrm{J}$ denotes the matrix of the exchange couplings and its elements are equal to $J_{1}$ if $(\mathrm{in})$ and $(\mathrm{jm})$ are nearest neighbors, to $J_{2}$ if they are next-nearest neighbors, and to zero otherwise. The $\delta^{n m}$ factor expresses the fact that interlayer dipolar couplings are neglected.

A mean-field calculation in our context amounts to diagonalizing $H$ and finding its lowest eigenvalue. The magnitude of the latter determines $T_{N}$ while the associated eigenvector defines the order parameter for the transition.29

Since $Q$ is diagonal in the layer indices we concentrate first on $J$. We define in-plane Fourier transforms $\mathbf{S}_{\mathbf{q} n}$ for the spins through:

$$
\mathbf{S}_{\mathbf{q} n}=\frac{1}{\sqrt{\mathcal{N}}} \sum_{i} \mathbf{S}_{i n} e^{i \mathbf{q} \cdot \mathbf{r}_{i}}
$$

where $\mathcal{N}$ is the number of spins per layer. Likewise we define $J(\mathbf{q}, m-n)$ as:

$$
J(\mathbf{q}, m-n)=\sum_{\mathbf{d}} J(\mathbf{d}, m-n) e^{i \mathbf{q} \cdot \mathbf{d}} .
$$

Since we expect the ordered phase to be homogeneous in the plane of the layers, we now restrict ourselves to the $\mathbf{q}=\mathbf{0}$ sector of the Hamiltonian. The neighbors and nearestneighbors of a spin in layer $n$ all belong to layers $n-1, n, n+1$. The only non-zero matrix elements of $J(\mathbf{0}, m-n)$ are thus:

$$
J(\mathbf{0}, 0)=6 J_{1} \text { and } J(\mathbf{0}, 1)=J(\mathbf{0},-1)=3\left(J_{1}+J_{2}\right) .
$$

The $\mathbf{q}=\mathbf{0}$ part of the Hamiltonian now reads:

$$
\mathcal{H}_{\mathbf{q}=\mathbf{0}}=\sum_{n m} A_{n m} \mathbf{S}_{\mathbf{0} n} \mathbf{S}_{\mathbf{0} m}
$$

where $A_{n m}=J(\mathbf{0}, m-n)$. Now $A$ can be diagonalized in the basis of $N$-dimensional orthonormal vectors $T_{k}$, whose components are:

$$
\left(T_{k}\right)_{n}=\sqrt{\frac{2}{N+1}} \sin \left(\frac{n k \pi}{N+1}\right)
$$

where $k$ is an integer ranging from 1 to $N$. The corresponding eigenvalues are: 


$$
a_{k}=6 J_{1}+6\left(J_{1}+J_{2}\right) \cos \left(\frac{k \pi}{N+1}\right) \text {. }
$$

In the same way as for $J$ in eq. (10) one can define a Fourier transform $Q_{\alpha \beta}(\mathbf{q})$. It is diagonal for $\mathbf{q}=\mathbf{0}$ with:

$$
\begin{array}{r}
Q_{x x}(\mathbf{0})=Q_{y y}(\mathbf{0})=-\mathcal{C} \\
Q_{z z}(\mathbf{0})=2 \mathcal{C}
\end{array}
$$

where

$$
\mathcal{C}=\frac{\left(g \mu_{b}\right)^{2}}{4} \sum_{i} \frac{1}{r_{i}^{3}}
$$

The lowest eigenvalue of matrix $H$ is thus $a_{N}-2 \mathcal{C}$ and the mean field $T_{N}$ is given by:

$$
\begin{aligned}
T_{N} & =-\frac{2 S^{2}}{3 k_{B}}\left(a_{N}-2 \mathcal{C}\right) \\
& =\frac{2 S^{2}}{3 k_{B}}\left(-6 J_{1}-6\left(J_{1}+J_{2}\right) \cos \left(\frac{N \pi}{N+1}\right)+2 \mathcal{C}\right) .
\end{aligned}
$$

Using bulk values for $J_{1}, J_{2}$ we find $T_{N}=6.93 \mathrm{~K}$. The associated order parameter is a linear combination of the in-plane projections of the spins with weights $\left(T_{k}\right)_{n}$ defined in (12):

$$
\mathbf{M}_{N}=\sqrt{\frac{2}{N+1}} \sum_{n} \sin \left(\frac{n N \pi}{N+1}\right) \mathcal{S}_{n}
$$

where $\mathcal{S}_{n}$ denotes the in-plane projection of the sum of all spins belonging to layer $n$. Note that, as $N \rightarrow \infty$ the usual antiferromagnetic staggered magnetization is recovered. We will in the next section check that $\mathbf{M}_{N}$ is indeed the correct order parameter.

\section{Monte Carlo simulation}

We have performed a Monte Carlo simulation for our system in order to check that dipolar interactions can drive a transition at a temperature $T_{N}>0$ and can generate an anisotropy in the susceptibility similar to that observed in experiments. We have also checked the relevance of the mean field order parameter. 
We present here results of a Monte Carlo simulation carried out on a system of 3 layers, each consisting of $23 \times 23$ spins, with periodic boundary conditions in the plane of the layers, in order not to introduce in-plane anisotropy. Each layer is thus mapped to a torus. The full Hamiltonian (7) has been used, where the distance $r_{i j}$ between pairs of sites has been taken to be the smallest distance on the torus between sites $i$ and $j$. All couplings in the Hamiltonian have been expressed in units of $J_{1}$ and we have used EuTe bulk values for the ratios $J_{2} / J_{1}=-3.75$ and $\left(g \mu_{b}\right)^{2} /\left(J_{1} a^{3}\right)=0.64$, which are the only parameters of our model. The heat-bath algorithm with sequential updating of the spins has been used, with 400 equilibration sweeps and 2000 sweeps with a measurement after each sweep. Error bars have been carefully computed as standard deviations of estimators of the observables. Three susceptibilities have been evaluated: $\chi_{z}$, along the normal to the layers, corresponding to $\chi_{\text {out }}$, and $\chi_{x}$ and $\chi_{y}$ in the plane of the layers. The specific heat has also been measured to check that it has a limit of $k_{B}$ per spin as $T \rightarrow 0$, a general property of classical spin systems 30 It is shown in Fig. 5 for the $\operatorname{EuTe}(3) / \operatorname{PbTe}(9)$ system. Susceptibilities have been computed as:

$$
\chi_{\alpha}=\frac{\left(g \mu_{b}\right)^{2}}{N \mathcal{N} k_{B} T}\left(\left\langle S_{\alpha}^{2}\right\rangle-\left\langle S_{\alpha}\right\rangle^{2}\right)
$$

where $\mathbf{S}$ is the total spin and $\mathcal{N}$ is the number of spins per layer.

Because the simulation has been done on a finite system, the in-plane rotational symmetry is not broken in the ordered phase with the result that the above averages are not well defined. We have thus evaluated $\chi_{x}$ and $\chi_{y}$ only at temperatures higher than the transition temperature.

Experimental and simulated susceptibilities have been plotted as a function of temperature in Fig. 6. One can see that the simulation qualitatively reproduces the anisotropy observed in the experiments, with a remarkable flatness of $\chi_{z}\left(\chi_{\text {out }}\right)$ at low temperature. We have also run simulations on 3 -layer systems of sizes $29 \times 29$ and $13 \times 13$ and these two systems didn't show any significant difference in the transition temperature or the low-temperature magnitude of $\chi_{z}$. 
We then checked the relevance of the order parameter $\mathbf{M}_{N}$ found in the mean field approach of section $\mathrm{B}$. In the same way as $\mathbf{M}_{N}$ in eq. (18) has been defined using vector $T_{k=N}$ each vector $T_{k}$ can be used to build an order parameter $\mathbf{M}_{k}$, defined by:

$$
\mathbf{M}_{k}=\sqrt{\frac{2}{N+1}} \sum_{n} \sin \left(\frac{n k \pi}{N+1}\right) \mathcal{S}_{n}
$$

using the same notations as before. The $\mathbf{M}_{k}$ 's are linear combinations of the in-plane projection of the spins. One could similarly define linear combinations of their $z$ components, although we know that spins order in-plane. For a 3-layer system, three order parameters can be defined:

$$
\begin{aligned}
& \mathbf{M}_{1}=\frac{1}{\sqrt{2}}\left(\frac{1}{\sqrt{2}} \mathcal{S}_{1}+\mathcal{S}_{2}+\frac{1}{\sqrt{2}} \mathcal{S}_{3}\right) \\
& \mathbf{M}_{2}=\frac{1}{\sqrt{2}}\left(\mathcal{S}_{1}-\mathcal{S}_{3}\right) \\
& \mathbf{M}_{3}=\frac{1}{\sqrt{2}}\left(\frac{1}{\sqrt{2}} \mathcal{S}_{1}-\mathcal{S}_{2}+\frac{1}{\sqrt{2}} \mathcal{S}_{3}\right) .
\end{aligned}
$$

One can define susceptibilities $\chi_{k}$ for the moduli of these three order parameters $k=1,2,3$ as:

$$
\chi_{k}=\frac{1}{N \mathcal{N} k_{B} T}\left(\left\langle\mathbf{M}_{k}^{2}\right\rangle-\left\langle\left|\mathbf{M}_{k}\right|\right\rangle^{2}\right)
$$

Because vectors $T_{k}$ are normalized, the $\chi_{k}$ all have the same leading behavior at high temperature. These susceptibilities are plotted in Fig.7. $\chi_{3}$ is the susceptibility that displays a sharp peak, thus suggesting that $\mathbf{M}_{3}$ is the appropriate order parameter for describing the phase transition.

\section{Low-temperature orthogonal susceptibility}

Since $\chi_{\text {out }}$ is flat at low temperature, it is desirable to have an estimate of its magnitude. We present here a calculation of $\chi_{\text {out }}$ using the spin Hamiltonian given in eq. (7).

$$
\mathcal{H}=\mathcal{H}_{e x}+\mathcal{H}_{d}
$$

where the exchange term is: 


$$
\mathcal{H}_{e x}=\sum_{i j, n m} J\left(\mathbf{r}_{j}-\mathbf{r}_{i}, m-n\right) \mathbf{S}_{i n} \cdot \mathbf{S}_{j m}
$$

and the dipolar term is:

$$
\mathcal{H}_{d}=\sum_{i j, n} Q_{\alpha \beta}\left(\mathbf{r}_{j}-\mathbf{r}_{i}\right) S_{i n}^{\alpha} S_{j n}^{\beta}
$$

In each layer we define a frame of reference as shown in Fig. 8, such that the $y$ axis lies along the direction of the layer magnetization, while the direction of the $z$ axis, orthogonal to the layers, is the same for all layers. The spin components $\sigma_{i n}^{\alpha}$ in the layer-dependent frames are related to the $S_{i n}^{\alpha}$ through:

$$
\begin{aligned}
& S_{i n}^{x}=(-1)^{n} \sigma_{i n}^{x} \\
& S_{i n}^{y}=(-1)^{n} \sigma_{i n}^{y} \\
& S_{i n}^{z}=\sigma_{i n}^{z} .
\end{aligned}
$$

At low temperature $\sigma_{i n}^{z}$ and $\sigma_{i n}^{x}$ will be small, while $\sigma_{i n}^{y}$ will be finite, with a fixed sign. $\chi_{\text {out }}$ is defined as:

$$
\chi_{\text {out }}=\frac{\left(g \mu_{b}\right)^{2}}{N \mathcal{N} k_{B} T}\left\langle\left(\sigma^{z}\right)^{2}\right\rangle
$$

where $\sigma^{z}=\sum_{i n} \sigma_{i n}^{z}$. For classical 3D spins $\left\langle\left(\sigma^{z}\right)^{2}\right\rangle$ is given by:

$$
\left\langle\left(\sigma^{z}\right)^{2}\right\rangle=\frac{1}{Z} \int \prod_{i n} \frac{d \sigma_{i n}^{z} d \sigma_{i n}^{x}}{S\left|\sigma_{i n}^{y}\right|}\left(\sigma^{z}\right)^{2} e^{-\beta \mathcal{H}}
$$

where $\beta=\frac{1}{k_{B} T}$. The partition function $Z$ reads:

$$
Z=\int \prod_{i n} \frac{d \sigma_{i n}^{z} d \sigma_{i n}^{x}}{S\left|\sigma_{i n}^{y}\right|} e^{-\beta \mathcal{H}}
$$

At low temperature $\left(\sigma_{i n}^{z}\right)^{2}$ and $\left(\sigma_{i n}^{x}\right)^{2}$ will be of order $k_{B} T$ if $\chi_{\text {out }}$ is to be finite. We can thus let the integrals run from $-\infty$ to $+\infty$, instead of $-S$ to $+S$. We note that $\sigma_{i n}^{y}$ appears both in the integration measure and $\mathcal{H}$ and is given by:

$$
\sigma_{i n}^{y}=\sqrt{S^{2}-\left({\sigma_{i n}^{z}}^{2}+{\sigma_{i n}^{2}}^{2}\right)}
$$

We now expand $\sigma_{i n}^{y}$ in powers of $\left({\sigma_{i n}^{z}}^{2}+{\sigma_{i n}^{x}}^{2}\right)$ keeping only the lowest order terms. This amounts to an expansion in powers of the temperature. The measure becomes $d \sigma_{i n}^{z} d \sigma_{i n}^{x} / S^{2}$. 
In the expression of $\beta \mathcal{H}$ only the first-order term need be kept. We are then left with a quadratic form for $\sigma_{i n}^{z}$ and $\sigma_{i n}^{x}$ which we need to diagonalize in order to calculate $\left\langle\left(\sigma^{z}\right)^{2}\right\rangle$.

Let us first consider $\mathcal{H}_{e x}$. In our approximation $\mathbf{S}_{i n} \cdot \mathbf{S}_{j m}$ reads:

$$
\mathbf{S}_{i n} \cdot \mathbf{S}_{j m}=\sigma_{i n}^{z} \sigma_{j m}^{z}+(-1)^{m-n}\left(\sigma_{i n}^{x} \sigma_{j m}^{x}+\left(S-\frac{\sigma_{i n}^{z}+\sigma_{i n}^{x}{ }^{2}}{2 S}\right)\left(S-\frac{{\sigma_{j m}^{z}}^{2}+\sigma_{j m}^{x}{ }^{2}}{2 S}\right)\right)
$$

After discarding constant terms, we get the following expression for $\mathcal{H}_{e x}$

$$
\mathcal{H}_{e x}=\sum_{i j, n m}\left(J\left(\mathbf{r}_{j}-\mathbf{r}_{i}, m-n\right)+\alpha_{n} \delta_{i j} \delta_{n m}\right)\left(\sigma_{i n}^{z} \sigma_{j m}^{z}+(-1)^{m-n} \sigma_{i n}^{x} \sigma_{j m}^{x}\right)
$$

where $\alpha_{n}=3\left(J_{2}-J_{1}\right)$ if $n=1$ or $n=N$ and $\alpha_{n}=6 J_{2}$ otherwise. Let us define Fourier transforms for $\sigma_{i n}^{z}, \sigma_{i n}^{x}$ and $J\left(\mathbf{r}_{j}-\mathbf{r}_{i}, m-n\right)$ in the same way as in (9) and (10). We now have:

$$
\mathcal{H}_{e x}=\sum_{n m, \mathbf{q}}\left(J(\mathbf{q}, m-n)+\alpha_{n} \delta_{n m}\right)\left({\sigma_{\mathbf{q} n}^{z}}^{*} \sigma_{\mathbf{q} m}^{z}+(-1)^{n} \sigma_{\mathbf{q} n}^{x}(-1)^{m} \sigma_{\mathbf{q} m}^{x}\right)
$$

Let us now turn to $\mathcal{H}_{d}$ and expand $\sigma_{i n}^{y}$. Because of the layer geometry $Q_{x z}=Q_{y z}=0$. Furthermore, since $Q(\mathbf{q}=\mathbf{0})$ is diagonal, non-diagonal terms $Q_{y x}\left(\mathbf{r}_{j}-\mathbf{r}_{i}\right) \sigma_{i n}^{y} \sigma_{j n}^{x}$ do not contribute at the quadratic order, but rather yield a term linear in $\sigma_{i n}^{x}$ which vanishes when summed on $j$. We are thus left with diagonal terms only. The contributions of $Q_{x x}$ and $Q_{z z}$ are then:

$$
\sum_{\mathbf{q} n} Q_{z z}(\mathbf{q})\left|\sigma_{\mathbf{q} n}^{z}\right|^{2}+Q_{y y}(\mathbf{q})\left|\sigma_{\mathbf{q} n}^{x}\right|^{2}
$$

and that of $Q_{y y}$, after expanding $\sigma_{i n}^{y}$ to first order, is:

$$
-Q_{y y}(\mathbf{q}=\mathbf{0}) \sum_{i}\left({\sigma_{i n}^{z}}^{2}+{\sigma_{i n}^{x}}^{2}\right)=\mathcal{C} \sum_{\mathbf{q} n}\left(\left|\sigma_{\mathbf{q} n}^{z}\right|^{2}+\left|\sigma_{\mathbf{q} n}^{x}\right|^{2}\right)
$$

Since only the $\mathbf{q}=\mathbf{0}$ mode contributes to $\sigma_{z}$, we now restrict $\mathcal{H}_{d}$ to its $\mathbf{q}=\mathbf{0}$ part. After using (14) and (15) we get

$$
\mathcal{H}_{d}=3 \mathcal{C} \sum_{n}{\sigma_{0 n}^{z}}^{2}
$$

and 


$$
\mathcal{H}_{e x}=\sum_{m n}\left(J(\mathbf{0}, m-n)+\alpha_{n} \delta_{n m}\right)\left(\sigma_{\mathbf{0} n}^{z} \sigma_{\mathbf{0} m}^{z}+(-1)^{n} \sigma_{\mathbf{0} n}^{x}(-1)^{m} \sigma_{\mathbf{0} m}^{x}\right) .
$$

We only need diagonalize the $z$ part of $\mathcal{H}$ which reads:

$$
\mathcal{H}_{z}=\sum_{n m} B_{n m} \sigma_{\mathbf{0} n}^{z} \sigma_{\mathbf{0} m}^{z}
$$

with

$$
B_{n m}=\left(3 \mathcal{C}+\alpha_{n}\right) \delta_{n m}+J(\mathbf{0}, m-n)
$$

Matrix $B$ has the same form as matrix $A$ used in section IVB with the difference that the matrix elements at both ends of the principal diagonal are different. B can be diagonalized in the basis of orthonormal vectors $U_{k}$ defined by:

$$
\left(U_{k}\right)_{n}=\frac{1}{N_{k}} \sin \left(\left(n-\frac{1}{2}\right) \frac{k \pi}{N}\right)
$$

where the integer $k$ ranges from 1 to $N$. The normalization factor $N_{k}$ is equal to $\sqrt{N}$ if $k=N$ and $\sqrt{N / 2}$ otherwise. The corresponding eigenvalues are:

$$
b_{k}=3 \mathcal{C}+6\left(J_{1}+J_{2}\right)\left(1+\cos \left(\frac{k \pi}{N}\right)\right) \text {. }
$$

Expanding $\sigma^{z}$ on the basis of the $\sigma_{\mathbf{0} k}^{z}$ defined by:

$$
\sigma_{\mathbf{0} k}^{z}=\sum_{n}\left(U_{k}\right)_{n} \sigma_{\mathbf{0} n}^{z}
$$

we get:

$$
\sigma^{z}=\sum_{k} \frac{\lambda_{k}}{N_{k}} \sigma_{0 k}^{z}
$$

where

$$
\lambda_{k}= \begin{cases}0 & \text { if } k \text { even } \\ \frac{\sqrt{\mathcal{N}}}{\sin \left(\frac{k \pi}{2 N}\right)} & \text { if } k \text { odd } .\end{cases}
$$

The integration over the $\sigma_{i n}^{z}$ in eq. (23) is straightforward since the $\sigma_{\mathbf{0} k}^{z}$ have Gaussian weights and we get the following result for $\chi_{\text {out }}$ at low temperature:

$$
\chi_{\text {out }}=\frac{\left(g \mu_{b}\right)^{2}}{2 N^{2}} \sum_{k=1}^{N} \frac{f_{k}}{3 \mathcal{C}+6\left(J_{1}+J_{2}\right)\left(1+\cos \left(\frac{k \pi}{N}\right)\right)}
$$


where the weights $f_{k}$ are given by:

$$
f_{k}= \begin{cases}0 & \text { if } k \text { even } \\ \frac{2}{\sin ^{2}\left(\frac{k \pi}{2 N}\right)} & \text { if } k \text { odd and } k \neq N \\ \frac{1}{\sin ^{2}\left(\frac{k \pi}{2 N}\right)} & \text { if } k \text { odd and } k=N\end{cases}
$$

The energy $\mathcal{C}$ is defined in (16) and equals $2.759 \frac{\left(g \mu_{b}\right)^{2}}{a^{3}}$. The $f_{k}$ satisfy the following sum rule: $\sum_{k} f_{k}=N^{2}$.

The $\chi_{\text {out }}$ we have found is temperature-independent; it is in fact the first term in an expansion of $\chi_{\text {out }}$ in powers of $T$. Using bulk values for the couplings, we find that for a 3-layer system $\chi_{\text {out }}=0.025 \frac{\left(g \mu_{b}\right)^{2}}{\left|J_{1}\right|}$ in excellent agreement with the simulation result in Fig. 6: $\left(0.026 \pm 8.10^{-4}\right) \frac{\left(g \mu_{b}\right)^{2}}{\left|J_{1}\right|}$. As the number of layers is increased, $\chi_{\text {out }}$ slowly decreases. For a 7-layer system, expression (35) yields: $\chi_{\text {out }}=0.017 \frac{\left(g \mu_{b}\right)^{2}}{\left|J_{1}\right|}$.

\section{DISCUSSION AND CONCLUSION}

The simulation results reported in section $\mathbb{I V C}$ for a 3-layer system qualitatively reproduce the anisotropy in susceptibilities observed in the experiments. However they do not agree quantitatively with the experimental results. We list below the experimental and theoretical values of $T_{N}$ and $\chi_{\text {out }}$ at low temperature, in dimensionless units.

$$
\begin{array}{lll}
T_{N}^{\text {exp }}=16.1 & T_{N}^{m c}=9.5 \pm 0.5 & \text { in units of } \frac{\left|J_{1}\right| S^{2}}{k} \\
\chi_{\text {out }}^{\text {exp }}=0.013 & \chi_{\text {out }}^{m c}=0.026 \pm 8.10^{-4} & \text { in units of } \frac{\left(g \mu_{b}\right)^{2}}{\left|J_{1}\right|}
\end{array}
$$

Our Monte Carlo simulations have been run on finite systems: although $T_{N}$ and $\chi_{\text {out }}$ didn't change appreciably when we increased the size of the system to $29 \times 29$ or decreased it to $13 \times 13$, we cannot rule out finite-size corrections. For $\chi_{\text {out }}$, however, analytic and simulation results are in excellent agreement which indicates that finite-size corrections are not significant in the low-temperature region. The numerical values listed above suggest that the effective value of $J_{1}$ in the samples is approximately twice as big as in the bulk. However, since the simulation uses bulk values for the ratios $J_{2} / J_{1}$ and $\left(g \mu_{b}\right)^{2} /\left(a^{3} J_{1}\right)$ this would imply 
that $J_{2}$ and the dipolar coupling are rescaled by the same factor. This cannot be the case, as the value of the dipolar coupling only depends on the in-plane lattice parameter, known from X-ray spectra. One possible explanation is that the exchange constants are different in the SL's relative to bulk values. Because of a $2.1 \%$ lattice mismatch between EuTe and PbTe, the SL will be strained and the in-plane and out-of-plane lattice constants will be different from one another, and different from their bulk values 14 . As a result, the exchange couplings will also be somewhat different. Within the family of Eu chalcogenides the lattice constant increases as the size of the anion increases from $\mathrm{O}$ to Te which makes it possible to study the dependence of the exchange couplings on the lattice parameter.7 In our case the in-plane lattice constant is reduced with respect to the bulk value while the out-of-plane constant is increased. Thus, in the SL's, the in-plane $J_{1}$ is likely to increase, while the out-of-plane $J_{1}$ will decrease.

Although dipolar interactions account for the flatness of $\chi_{\text {out }}$ at low temperature, we can expect a single-ion anisotropy term of the form $\kappa \sum_{i n} S_{i n}^{z}{ }^{2}$ to have the same effect. Let us replace $\mathcal{H}_{d}$ with such a term. The calculation of $\chi_{\text {out }}$ is similar, except that in equation (28), $3 \mathcal{C}$ is replaced by $\kappa$. Thus for single-ion anisotropy to have the same effect as dipole interactions, $\kappa$ would have to be of the order of $0.2 \mathrm{~K}$, which is larger than the exchange couplings, an unlikely situation.

In conclusion, we have presented experimental susceptibilities of EuTe/PbTe short-period antiferromagnetic superlattices. We suggest that dipolar interactions may stabilize longrange order in these $2 \mathrm{D}$ structures. Additional theoretical work along with more precise susceptibility data and specific heat measurements are needed to confirm this hypothesis and to study the critical behavior, an aspect not touched upon in the present work.

\section{ACKNOWLEDGEMENTS}

The authors are indebted to Profs M.S. and G. Dresselhaus, A. Billoire, Th. Jolicour for many discussions and suggestions. They wish to thank Profs G. Springholz and G. Bauer for 
making the samples used in the present study available and are grateful to the Pittsburgh Supercomputing Center for a computing time allocation under grant no. DMR-95-0022P. L.B. acknowledges support from NATO under Research Grant no. 3B94FR and wishes to thank Prof. M.S. Dresselhaus and MIT for their hospitality as well as CEA for its support. Research at MIT was supported in part by NSF grant DMR-95-10093. 


\section{REFERENCES}

${ }^{1}$ R. F. C. Farrow, in Magnetism and Structure in Systems of Reduced Dimensions, (Plenum Press New York, 1993), Vol. 309. NATO ASI series B.

${ }^{2}$ Ultrathin Magnetic Structures, edited by J. A. C. Bland and B. Heinrich (Springer-Verlag, New York, 1994).

${ }^{3} \mathrm{P}$. Wachter, in Handbook on the Physics and Chemistry of Rare Earths, (North-Holland Publishing Company, Amsterdam, 1979).

${ }^{4}$ A. Mauger and C. Godart, Phys. Rep. 141, Nos. 2\&3, 51 (1986).

${ }^{5}$ P. K. Streit and G. E. Everett, Phys. Rev. B 21, 169 (1980).

${ }^{6}$ J. W. Battles and G. E. Everett, Phys. Rev. B 1, 3021 (1970).

${ }^{7}$ W. Zinn, J. Magn. Magn. Mater. 3, 23 (1976).

${ }^{8}$ G. Will, S. J. Pickart, A. Alperin, and R. Nathans, Phys. Chem. Solids 24, 1679 (1963).

9 J. I. Kaplan, J. Chem. Phys. 22, 1709 (1954).

${ }^{10}$ F. Keffer and W. O’Sullivan, Phys. Rev. 108, 637 (1957).

${ }^{11}$ G. Springholz and G. Bauer, Phys. Rev. B 48, 10998 (1993).

${ }^{12}$ G. Springholz and G. Bauer, J. Appl. Phys. 77, 540 (1995).

${ }^{13}$ G. Springholz, N. Frank, and G. Bauer, Appl. Phys. Lett. 64, 2970 (1994).

14 J.J. Chen, G.Dresselhaus, M.S. Dresselhaus, G. Springholz, C.Pichler, and G. Bauer, Phys. Rev. B 54, 402 (1996).

${ }^{15}$ Z. Wilamowski W. Jantsch and G. Springholz, Materials Science Forum 182-184, 669 (1995).

16 J.J. Chen, PhD thesis, MIT, (1996). 
${ }^{17}$ N. Frank, G. Springholz, and G. Bauer, Phys. Rev. Lett. 73, 2236 (1994).

${ }^{18}$ M. Shima, L. Salamanca-Riba, G. Springholz, and G. Bauer, Symposium on the Evolution of Epitaxial Structure and Morphology, Fall MRS (1995).

19 T. M. Giebultowicz, V. Nunez, G. Springholz, G. Bauer, J. Chen, M.S. Dresselhaus, and J.K. Furdyna, J. Magn. Magn. Mat. 140-144, 635 (1995).

${ }^{20}$ N. D. Mermin and H. Wagner, Phys. Rev. Lett. 17, 1133 (1966).

${ }^{21}$ D. Pescia and V. L. Pokrovsky, Phys. Rev. Lett. 65, 2599 (1990).

${ }^{22}$ R. P. Erickson and D. L. Mills, Phys. Rev. B 43, 10715 (1991).

${ }^{23}$ S. V. Maleev, Zh. Eksp. Theor. Fiz. 70, 2374 (1976) [Sov. Phys. JETP 43, 1240 (1976)].

${ }^{24}$ V. L. Pokrovskii and M. V. Feigel'man, Zh. Eksp. Teor. Fiz. 72, 557 (1977) [Sov. Phys. JETP 45, 291 (1977)].

${ }^{25}$ S. T. Chui, Phys. Rev. B 50, 12559 (1994).

${ }^{26}$ C. Pich and F. Schwabl, Phys. Rev. B 49, 413 (1994).

${ }^{27}$ A. S. de Arruda, N. Majlis, S. Selzer, and W. Figueiredo, Phys. Rev. B 51, 3933 (1995).

${ }^{28}$ H. Benson and D. L. Mills, Phys. Rev. 178, 839 (1969).

${ }^{29}$ See for example chapter 4 of: Quantum Many-particle Systems, by John W. Negele and Henri Orland, (Addison Wesley, 1988).

${ }^{30}$ M. E. Fisher, Am. J. Phys. 32, 343 (1964). 


\section{FIGURES}

(a)

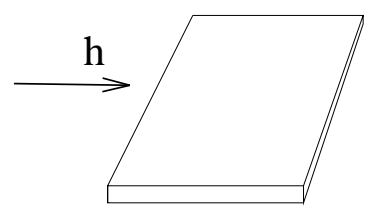

$\chi_{\text {in }}$ (b)

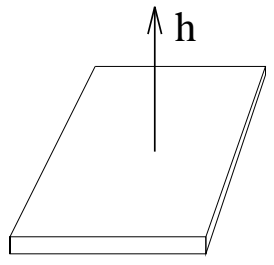

Xout

FIG. 1. The two principal orientations of the sample with respect to the AC probing field for the $\chi_{\text {in }}(\mathrm{a})$, and $\chi_{\text {out }}$ (b) susceptibility measurements. 

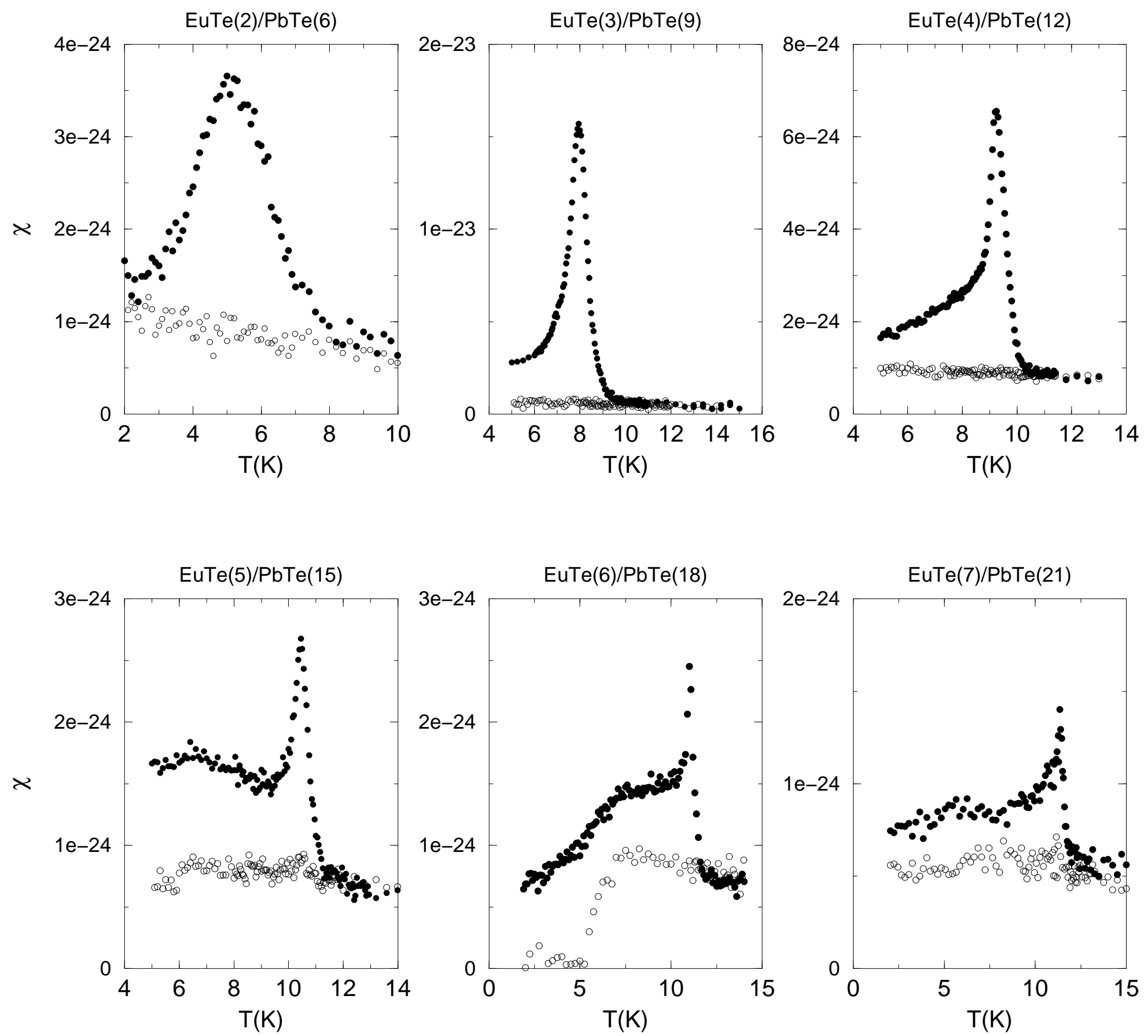

FIG. 2. $\chi_{\text {in }}$ (in-plane, $\bullet$ ) and $\chi_{\text {out }}$ (orthogonal, ○) susceptibilities in emu normalized per Eu atom, for samples $\mathrm{EuTe}(2) / \mathrm{PbTe}(6)$ through $\mathrm{EuTe}(7) \mathrm{PbTe}(21)$. 


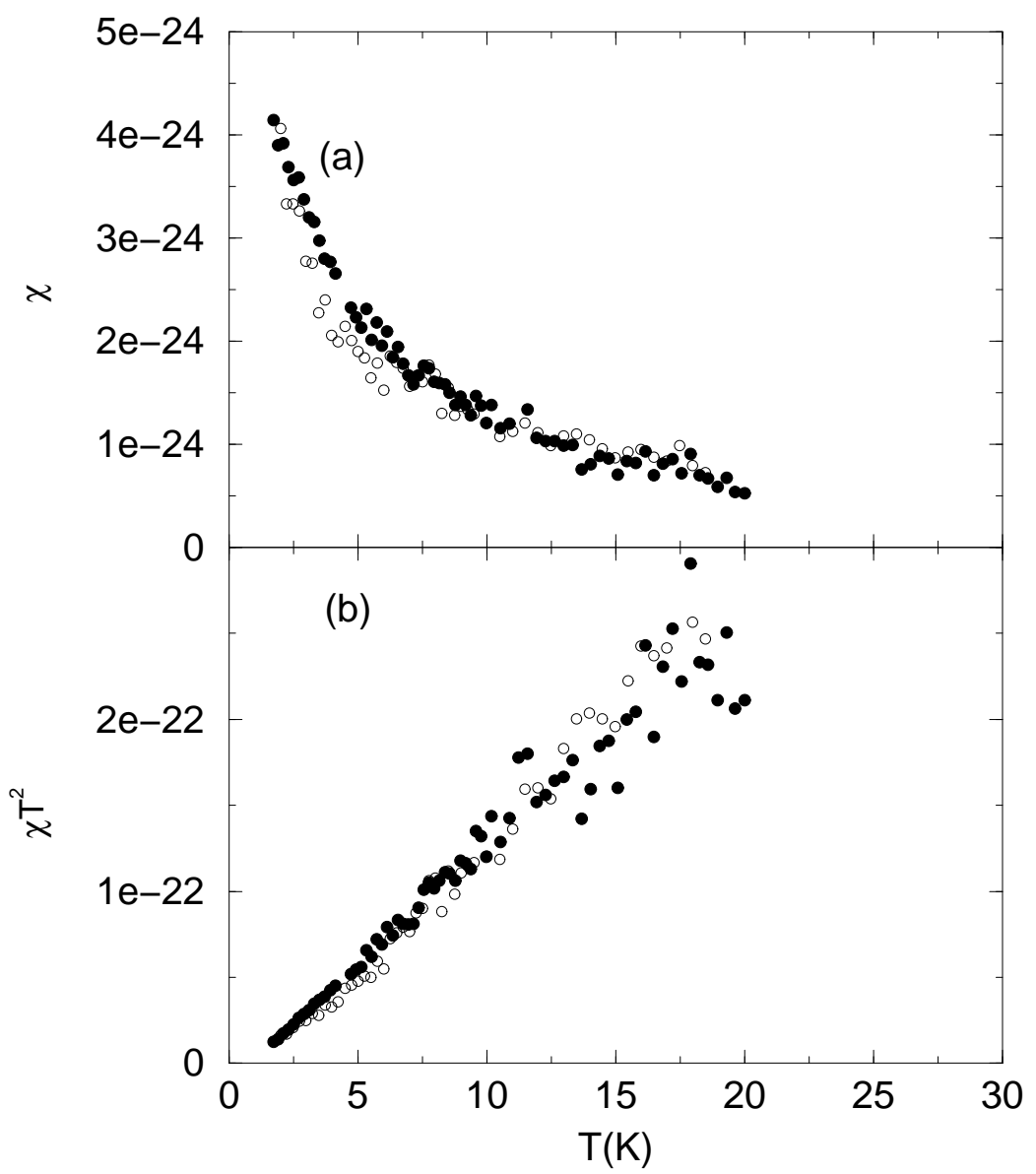

FIG. 3. (a) $\chi_{\text {in }}\left(\right.$ in-plane, $\bullet$ ) and $\chi_{\text {out }}($ orthogonal, o) susceptibilities in emu normalized per Eu atom, for sample EuTe(1)PbTe(3). (b) Same susceptibilities, multiplied by $T^{2}$. 


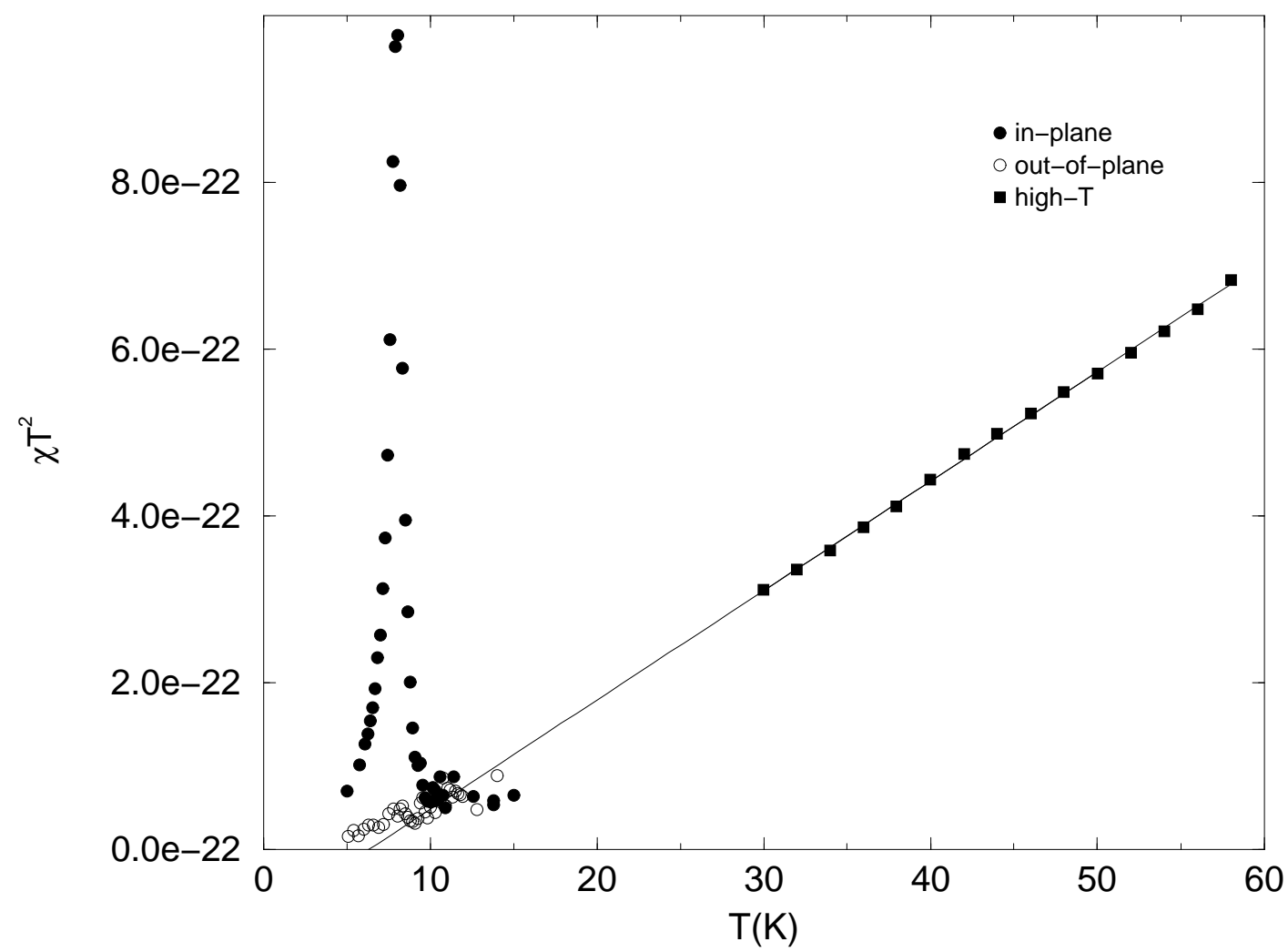

FIG. 4. A plot of $\chi T^{2}$ for sample $\operatorname{EuTe}(3) / \operatorname{PbTe}(9)$ in the paramagnetic phase (squares) and in the ordered phase, in the in-plane $(\bullet)$ and out-of-plane (०) directions, in emu normalized per Eu atom. 


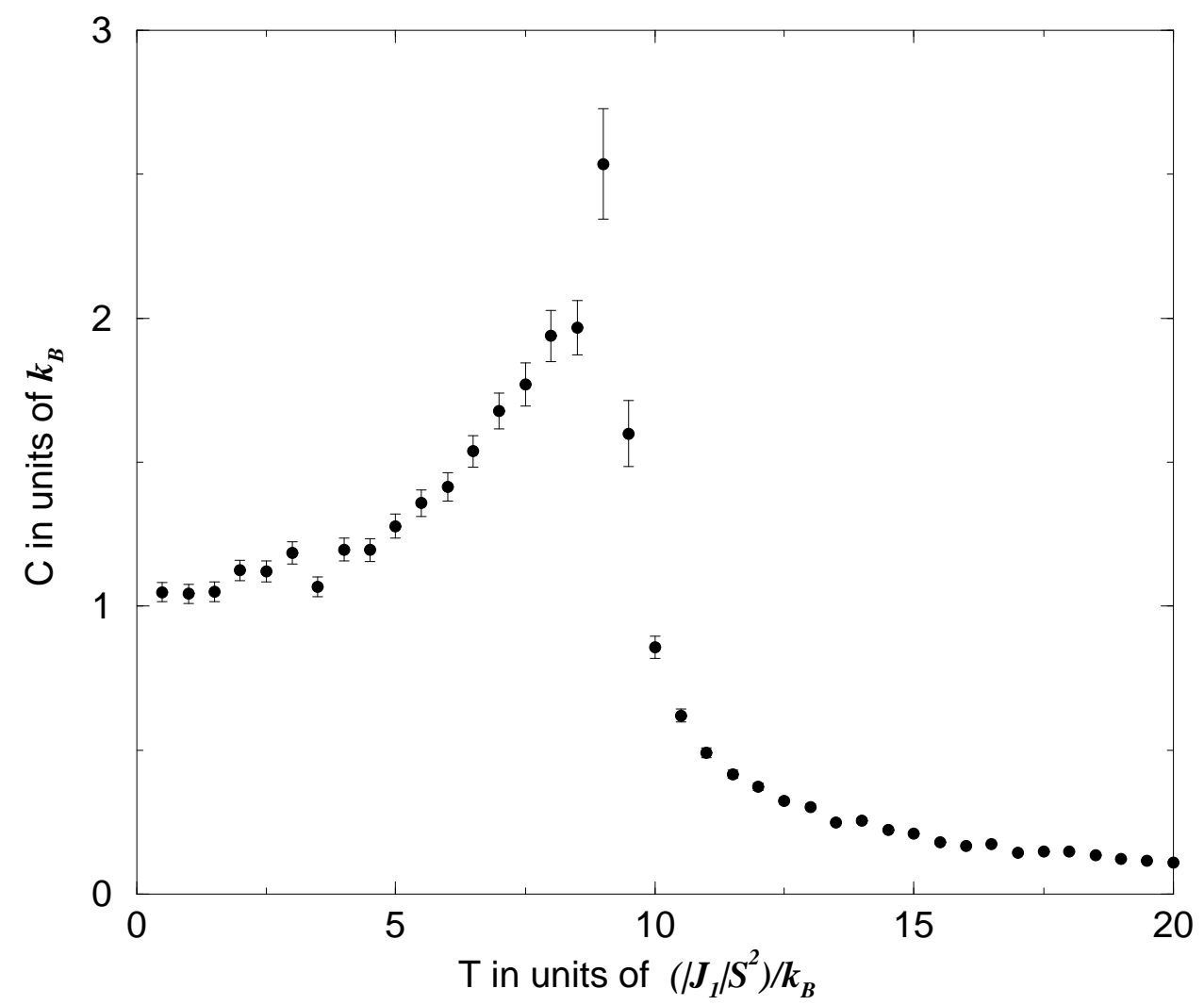

FIG. 5. Simulated specific heat for a $\operatorname{EuTe}(3) / \operatorname{PbTe}(9)$ system in units of $k_{B}$. 

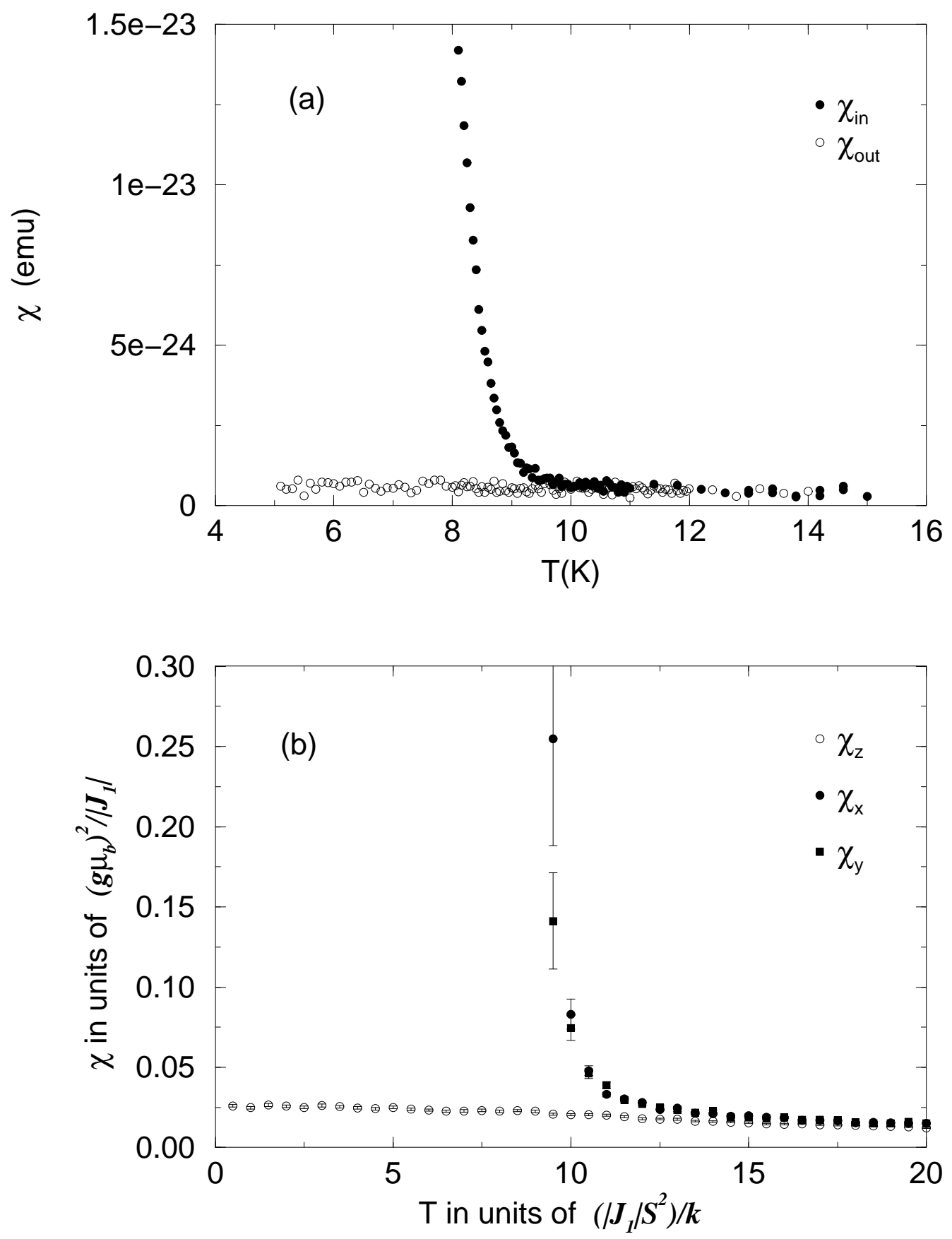

FIG. 6. (a) Measured $\chi_{\text {in }}$ and $\chi_{\text {out }}$ for the $\operatorname{EuTe}(3) / \operatorname{PbTe}(9)$ sample. (b) Simulated $x, y, z$ susceptibilities for a 3 -layer system of size $23 \times 23$. 


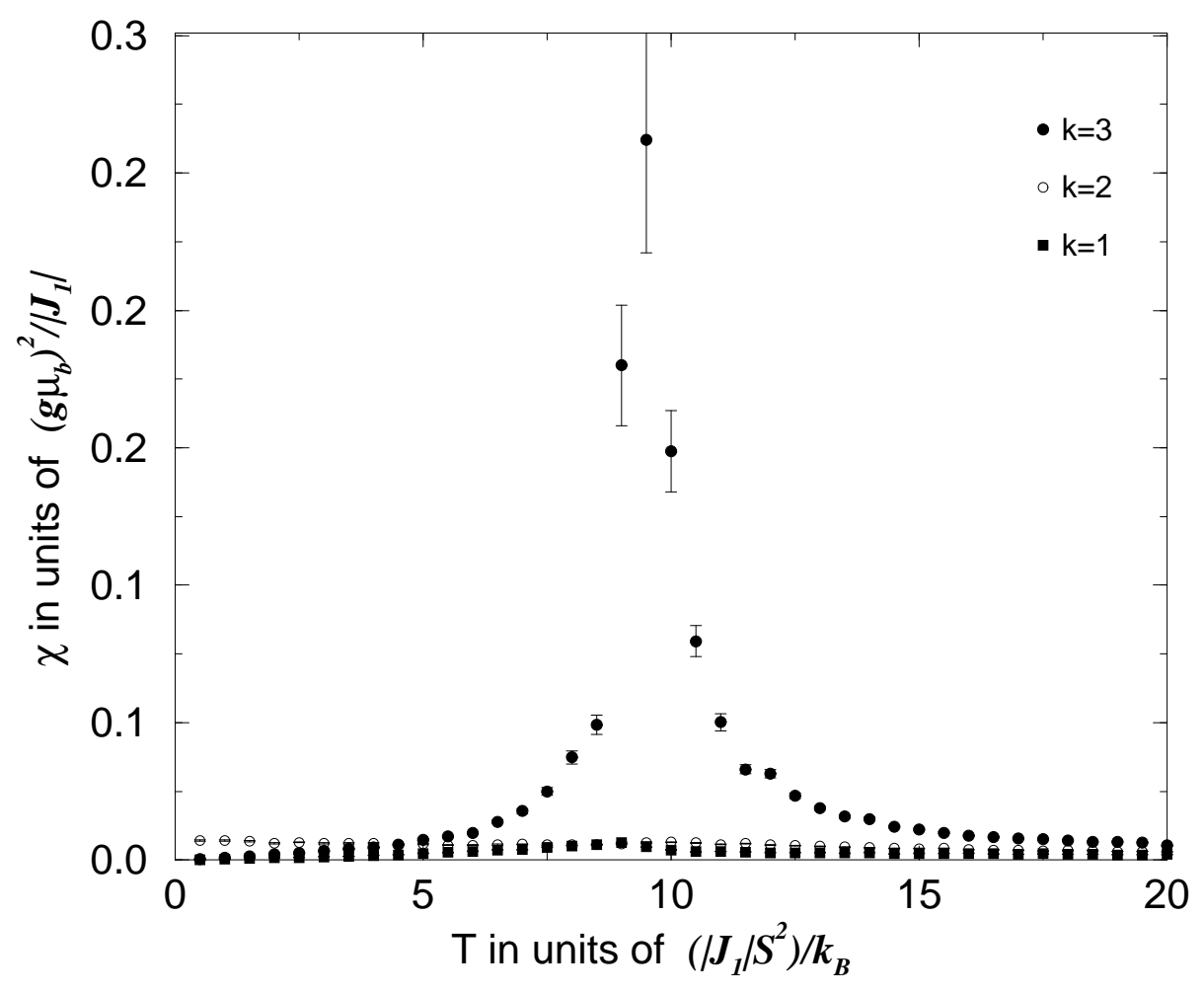

FIG. 7. Simulated susceptibilities corresponding to order parameters $\mathbf{M}_{k}$, for a 3-layer system of size $23 \times 23$. 

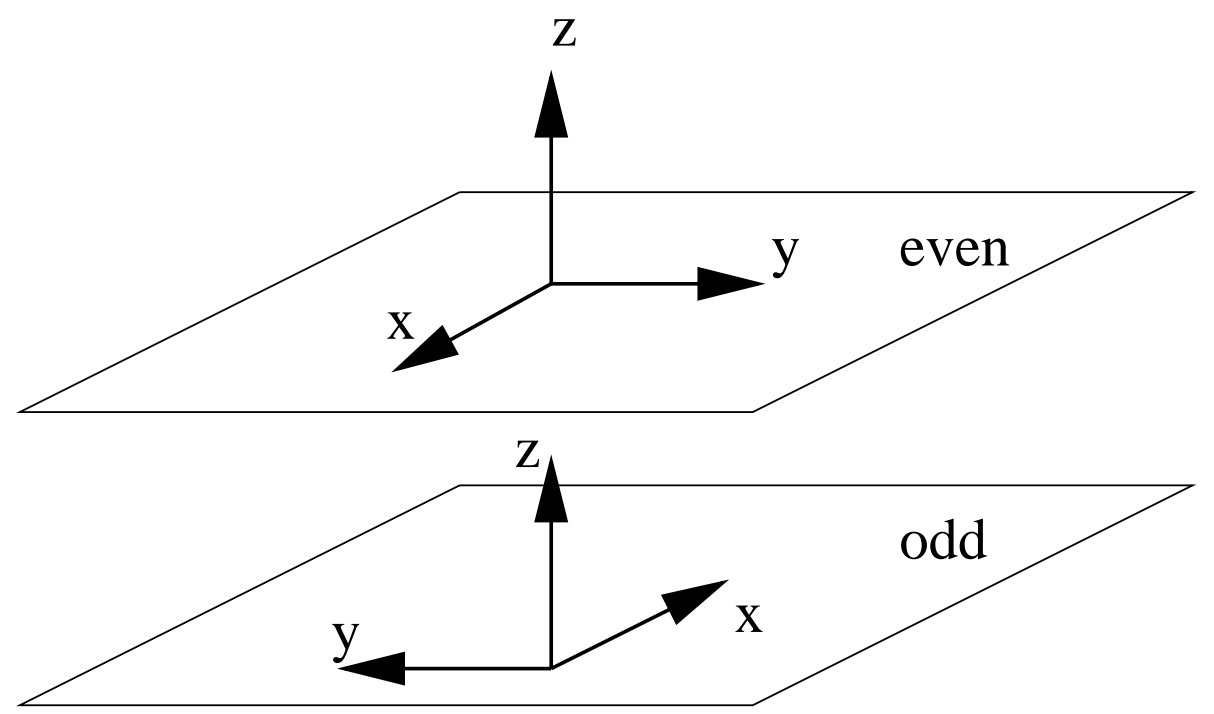

FIG. 8. Layer-dependent reference frames as defined in section IV. D. 